Results 44\% preferred the option of both appointments and drop in, whilst $28 \%$ each favoured either all appointments or drop in access only. There was no preferred time of attendance. Discussion/conclusion As the service already provides both appointments and drop in access the audit provided little to no evidence that a change to service delivery would reduce levels of non-attendance. There remains minimal data about how best to fulfil public and individual sexual health obligations, especially to an extensive rural community such as ours. A further audit on actual non-attenders could identify patterns in patient expectation.

\section{P140 MISSED OPPORTUNITIES FOR ENSURING ADEQUATE CONTRACEPTION: LESSONS FROM A RURAL SEXUAL HEALTH SERVICE}

Lena Budd, Amy Pearce, Frances Keane, George Morris*. Sexual Health Hub, Royal Cornwall Hospital, Truro, Cornwall, UK

\subsection{6/sextrans-2015-052126.183}

Background/introduction Our county-wide service is undergoing increasing integration which makes public health sense. Ideally, risk of both sexually transmitted infections and pregnancy should be addressed with patients.

Aim(s)/objectives We looked at missed opportunities for ensuring adequate contraception during routine $\mathrm{GU}$ appointments.

Methods A retrospective notes review of 50 consecutive new female attendances over 2/12 was conducted, with a follow up at $4 / 12$ to check contraception initiation or pregnancy.

Results Consultations were conducted by 16 different staff, $44 \%(7)$ of whom are trained to initiate oral contraceptive pills (OCPs), 4 fit implants and 2 fit IUCD/IUS. 23 and 27 patients were seen by nurses and doctors respectively. Contraception methods, including none, were universally documented. 22(44\%) patients were using long acting reversible methods of contraception (LARC) and 28\%(14) an OCP. Pill compliance was documented in 5(36\%) and advice given in 1 case. Only 4 (14\%) of the 28 non -LARC patients had LARC discussion. 7 patients used condoms and 7 no contraception. 5(36\%) of these were advised to book a contraception clinic (CC)/GP appointment for contraception, 2 of whom failed to attend a subsequent CC. 1 patient was quick- started on an OCP. 2 patients were known to have conceived during the subsequent 4/12; 1 had LARC and 1 OCP at initial visit. 6(12\%) and 1 patient/s were deemed at risk of pregnancy and appropriately provided with emergency contraception respectively.

Discussion/conclusion There were missed opportunities to maximise contraception efficacy. Time restrictions and lack of staff training pose barriers which we need to address.

\section{P141 HOW ACCURATE IS CLINICAL CODING IN RECENTLY INTEGRATED SEXUAL HEALTH SERVICES?}

Athavan Umaipalan, Laura Parry, Lalitha Kiritharan, Heather Anderson, Liat Sarner*, Margaret Portman. Barts Health NHS Trust, London, UK

\subsection{6/sextrans-2015-052126.184}

Background/introduction Clinical coding in England provides monitoring data for Public Health England via the Genitourinary Medicine Clinic Activity Dataset (GUMCAD) and Sexual and
Reproductive Health Activity (SRHAD) returns. In London, this data is also used to reflect activity for the Integrated Sexual Health Tariff (ISHT) which may form the basis for payment in future. Integration of contraception and GUM services presents a challenge in maintaining accuracy of clinical coding.

Aim(s)/objectives To audit the accuracy of SHAPPT, SRHAD and SRH coding in a multi-site integrated sexual health service, comparing sites traditionally providing GUM services vs contraception.

Methods Local standards were agreed; 95\% of patients should have accurate SHAPPT, SRHAD and SRH codes. 229 records from 2 GUM sites and 53 from 1 contraception site were audited from attendances between May and July 2014.

Results

\begin{tabular}{lll}
\hline & $\begin{array}{l}\text { Traditional GUM } \\
\text { (\% correct) }\end{array}$ & $\begin{array}{l}\text { Traditional contraception } \\
\text { (\% correct) }\end{array}$ \\
\hline "T" codes & $140 / 142(99 \%)$ & $22 / 25(88 \%)$ \\
P1A, P1B, P1C codes & $209 / 229(91 \%)$ & $7 / 34(21 \%)$ \\
A-C codes & $58 / 67(86.5 \%)$ & $3 / 11(27 \%)$ \\
SRHAD & $31 / 46(67 \%)$ & $29 / 31(94 \%)$ \\
SRH & $2 / 20(10 \%)$ & $5 / 9(55 \%)$ \\
\hline
\end{tabular}

Discussion/conclusion As expected, the accuracy of coding reflected the traditional nature of the sites. The locally set standard of $95 \%$ was only reached on one occasion. Missing SRH codes alone would equate to lost income of $£ 1259$ from 77 visits if the ISHT was in place. Staff training and weekly capture and correction of missing HIV codes through targeted email reminders has resulted in an improvement in coding.

\section{P142 USING THE "SPOTTING THE SIGNS" PROFORMA IN A GUM CLINIC TO FACILITATE IDENTIFICATION OF CHILD SAFEGUARDING CONCERNS}

Joanne Pye*, Nadi Gupta. Rotherham District General Hospital, Rotherham, South Yorkshire, UK

\subsection{6/sextrans-2015-052126.185}

Background/introduction In the wake of recent events regarding child sexual exploitation, BASHH produced the 'Spotting the Signs' guidance. Our GUM department has been using the 'Spotting the Signs' proforma since August 2014 for all under 16 year olds routinely and any patients aged 16-17 where concerns identified.

Aim(s)/objectives The aim of this project was to review the data gathered using the proforma and review the number of safeguarding referrals made.

Methods All under $16 \mathrm{~s}$ and any patients aged $16-17$ seen between August and December 2014 were identified. A retrospective case note review was undertaken of all the proformas. Data gathered included non-consensual sex, age differences, drug and alcohol issues, coercion and number of referrals to child safeguarding.

Results 20 patients were identified (16 female, 4 male); 18 cases were under 16 years. Two patients aged $16-17$ had been assessed using the proforma. $50 \%$ of patients were identified as having mental health issues, 55\% were identified with concerns regarding exploitation and $20 \%$ were noted to have problematic drug/ alcohol use. $55 \%$ of patients were referred to safeguarding services. 
Discussion/conclusion Use of the proforma has increased identification of mental health issues, highlighted concerns regarding age differences and provided details of drug/alcohol use, social circumstances and sexual exploitation. The data suggests that use of the proforma allows a more detailed risk assessment thereby increasing the likelihood of identifying safeguarding issues. We initially used the proforma routinely in all under 16 year olds and have since expanded this to all under 18 year olds.

\section{P143 IMPLEMENTATION OF AN ASYMPTOMATIC PATHWAY SIGNIFICANTLY REDUCES CLINIC VISIT DURATION}

Sarah Cochrane*, Helen Wheeler, Lindsey Harryman. Bristol Sexual Health Centre, Bristol, UK

\subsection{6/sextrans-2015-052126.186}

Background/introduction Our sexual health clinic in a busy citycentre is experiencing increasing patient demand. The challenge is to provide time-efficient, quality patient-care. Developing a structured screening pathway for asymptomatic patients to be seen by nursing assistants (NAs) could reduce time spent within clinic.

Aim(s)/objectives

1. To successfully and safely introduce a pathway enabling NAs to screen asymptomatic, heterosexual patients.

2. To assess the pathway's impact on patient-care including:

- Time spent within clinic

- Screening tests offered/accepted (following BASHH guidance)

Methods

- Baseline data was recorded for two weeks prior to pathway introduction.

- The asymptomatic pathway was implemented, including selfcompleted symptom questionnaire and patient assessment/testing tool.

- A competency package for NAs was introduced.

- Comparison of patient-care to baseline was made.

Results Eighty asymptomatic patients were identified during the initial two-week period. Following introduction, thirty-three patients followed the pathway. Four subsequently disclosed symptoms and were excluded.

\begin{tabular}{llll} 
Abstract P143 Table 1 & \multicolumn{3}{l}{ Asymptomatic pathway } \\
\hline & $\begin{array}{l}\text { Pre Asymptomatic } \\
\text { Pathway }\end{array}$ & $\begin{array}{l}\text { Post Asymptomatic } \\
\text { Pathway }\end{array}$ \\
& $\mathbf{( 8 0}$ patients) & (29 patients) & p Value \\
\hline Mean Time in clinic (minutes) & 67 & 44 & 0.00001 \\
HIV Testing Offered & $79(98.7 \%)$ & $29(100 \%)$ & 0.55 \\
HIV Testing Accepted & $66(83.5 \%)$ & $25(86 \%)$ & 0.65 \\
Chlamydia positive NAATs & $2(2.5 \%)$ & $0(0 \%)$ & 0.39 \\
\hline
\end{tabular}

Discussion/conclusion Early results show significant reductions in clinic visit duration. This improves patient experience, increases patient numbers and allows trained staff to manage complex patients. HIV test offer and uptake increased. More data are needed for future analyses. NAs will continue to be supported in pathway provision. Further elements will be introduced to assess and manage risk-taking behaviour.

\section{P144 VALIDATION OF THE DENVER HIV RISK SCORE FOR TARGETING HIV SCREENING IN VANCOUVER, BRITISH COLUMBIA}

${ }^{1}$ Titilola Falasinnu*, ${ }^{1}$ Paul Gustafson, ${ }^{2}$ Mark Gilbert, ${ }^{1}$ Jean Shoveller. ${ }^{1}$ University of British Columbia, Vancouver, Canada; ${ }^{2}$ British Columbia Centre for Disease Control, Vancouver, Canada

\subsection{6/sextrans-2015-052126.187}

Background/introduction The Denver HIV risk score (DHRS) is a prediction rule developed for targeting HIV testing and validated in U.S. clinical settings (PMID: 22431561). The final model of the DHRS included age, gender, race/ethnicity, sex with a male, vaginal intercourse, receptive anal intercourse, injection drug use, and past HIV testing.

Aim(s)/objectives We aimed to validate the DHRS in patients attending two publicly funded STI clinics in Vancouver, British Columbia.

Methods We validated the model using electronic records (2000-2012) from 47,175 clinic visits. Each visit was scored based on variables included in the DHRS. Visits were stratified into 5 risk groups according to their score: very low $(<20)$, low (20-29), moderate (30-39), high (40-49), and very high $(\geq 50)$. The model's discrimination and calibration for predicting an HIV diagnosis were examined by AUC and the Hosmer-Lemeshow (H-L) statistic. We examined the sensitivity and proportion of patients that would need to be screened at different cutoffs of the risk score.

Results The prevalence of HIV infection was $0.46 \%$. Validation demonstrated good performance: the AUC was $0.80 \quad(95 \%$ CI: $0.79-0.81)$ and the H-L $\chi^{2}=8.8,8 \mathrm{df}, p=0.36$. HIV prevalence within each risk groups was: $0 \%, 0.05 \%, 0.25 \%, 0.86 \%$, and $1.23 \%$, respectively. HIV testing is recommended for scores of $\geq 40$. The DHRS identified cases with a sensitivity of $96 \%$ and a fraction screened of $41 \%$.

Discussion/conclusion The DHRS performed well in these STI clinic settings in Vancouver, accurately identifying individuals at increased HIV risk, and may be useful for providing individualised estimates of risk as part of routine HIV screening.

\section{P145 INTRODUCTION AND TRIAL OF A "CHEMSEX" SUPPORT SERVICE IN A SOUTH WEST LONDON GU CLINIC}

${ }^{1}$ Bavithra Nathan*, ${ }^{2}$ Julia Waters, ${ }^{1}$ Richard Simms, ${ }^{1}$ Simon Green, ${ }^{3}$ Clare Husbands, ${ }^{1}$ Graham Milgrew, ${ }^{1}$ Gill McCarthy. ${ }^{1}$ Kingston Hospital NHS Foundation Trust, Kingston Upon Thames, UK; ${ }^{2}$ Public Health Directorate, Royal Borough of Kingston-Upon-Thames, UK; ${ }^{3}$ Kingston Wellbeing Service, CNWL - Central \& North West London NHS Foundation Trust, UK

\subsection{6/sextrans-2015-052126.188}

Background/introduction Since 2013 our centre has recognised a problem of recreational use drug associated with sex amongst MSM.

Aim(s)/objectives A joint survey with the local commissioners was set up to establish the extent of the problem in the borough and to identify a need for further services.

Methods 100 HIV negative MSM and 50 HIV positive MSM completed a patient survey with questions regarding recreational drug use related to "chemsex".

Results Results indicated a high level of drug use with $60 \%$ $(90 / 150)$ reporting any drug use and 21\% (32/150) specifically using party drugs in the last 6 months. Clients were asked where they would like to have a specialist drug service and the 